\title{
Acute-on-chronic liver failure alters meropenem pharmacokinetics in critically ill patients with continuous hemodialysis: an observational study
}

Jörn Grensemann ${ }^{1 *}$, David Busse ${ }^{2,3}$, Christina König ${ }^{1,4}$, Kevin Roedl ${ }^{1}$, Walter Jäger ${ }^{5}$, Dominik Jarczak , Stefanie Iwersen-Bergmann ${ }^{6}$, Carolin Manthey ${ }^{7}$, Stefan Kluge ${ }^{1}$, Charlotte Kloft ${ }^{2 \dagger}$ and Valentin Fuhrmann ${ }^{1,8+}$

\begin{abstract}
Background: Infection and sepsis are a main cause of acute-on-chronic liver failure (ACLF). Adequate dosing of antimicrobial therapy is of central importance to improve outcome. Liver failure may alter antibiotic drug concentrations via changes of drug distribution and elimination. We studied the pharmacokinetics of meropenem in critically ill patients with ACLF during continuous veno-venous hemodialysis (CVVHD) and compared it to critically ill patients without concomitant liver failure (NLF).

Methods: In this prospective cohort study, patients received meropenem $1 \mathrm{~g}$ tid short-term infusion (SI). Meropenem serum samples were analyzed by high-performance liquid chromatography. A population pharmacokinetic analysis was performed followed by Monte Carlo simulations of (A) meropenem $1 \mathrm{~g}$ tid Sl, (B) $2 \mathrm{~g}$ loading plus $1 \mathrm{~g}$ prolonged infusion tid (C) $2 \mathrm{~g}$ tid Sl, and (D) $2 \mathrm{~g}$ loading and continuous infusion of $3 \mathrm{~g} /$ day on days 1 and 7. Probability of target attainment (PTA) was assessed for $4 \times$ the epidemiological cut-off values for Enterobacterales $(4 \times 0.25 \mathrm{mg} / \mathrm{L})$ and Pseudomonas spp. $(4 \times 2 \mathrm{mg} / \mathrm{L})$.

Results: Nineteen patients were included in this study. Of these, 8 patients suffered from ACLF. A two-compartment model with linear clearance from the central compartment described meropenem pharmacokinetics. The peripheral volume of distribution $\left(V_{2}\right)$ was significantly higher in ACLF compared to NLF (38.6L versus 19.7L, $p=.05$ ). PTA for Enterobacterales was achieved in 100\% for all dosing regimens. PTA for Pseudomonas spp. in ACLF on day 1/7 was: A: 18\%/80\%, B: 94\%/88\%, C: 85\%/98\% D: 100\%/100\% and NLF: A: 48\%/65\%, B: 91\%/83\%, C: 91\%/93\%, D: 100\%/100\%.

Conclusion: ALCF patients receiving CVVHD had a higher $V_{2}$ and may require a higher loading dose of meropenem. For Pseudomonas, high doses or continuous infusion are required to reach PTA in ACLF patients.

Keywords: Antibiotics, Target attainment, Intensive care, Volume of distribution, Monte Carlo simulation, Population pharmacokinetics, Probability of target attainment
\end{abstract}

*Correspondence: j.grensemann@uke.de

${ }^{\dagger}$ Charlotte Kloft and Valentin Fuhrmann are shared senior authors ${ }^{1}$ Department of Intensive Care Medicine, University Medical Center Hamburg-Eppendorf, Martinistraße 52, 20246 Hamburg, Germany Full list of author information is available at the end of the article

\section{Background}

Sepsis and septic shock are frequent complications in patients with acute-on-chronic liver failure (ACLF) and associated with a high mortality [1-7]. Early empiric broad-spectrum antibiotic therapy is indicated in these critically ill patients [8]. Meropenem is often used for this purpose due to its broad spectrum and still favorable 
resistance profile. Acute kidney injury is the most frequent type of organ failure in ACLF [3] requiring renal replacement therapy in this patient population [9]. Attaining sufficient antibiotic concentrations is important for the therapy of sepsis. Due to an increased volume of distribution $(V)$ as a result of capillary leak syndrome and a decreased elimination due to organ dysfunction, antibiotic pharmacokinetics (PK) is highly variable in critical illness. Therefore, dosing recommendations for meropenem during renal replacement therapies range from 0.5 to $3 \mathrm{~g}$ per day [10]. Concerning hepatic insufficiency, only PK and pharmacodynamic data from patients with stable alcoholic cirrhosis are available [11], but to date, there are no available data in patients suffering from ACLF with multiorgan failure.

Therefore, we studied the impact of ACLF on PK of meropenem in critically ill patients requiring continuous veno-venous hemodialysis (CVVHD) in comparison to critically ill patients receiving CVVHD without ACLF.

\section{Methods}

\section{Ethics}

The study was approved by the Ethics Committee of the Hamburg Chamber of Physicians, Germany (Reference: PV5415). Consent was obtained from the patients' closest relatives or legal surrogates.

\section{Study design}

Patients eligible for this open-label observational prospective cohort study were receiving meropenem for clinical indication and required CVVHD. Patients $<18$ years or with an extracorporeal circuit other than the CVVHD were excluded. Patients were grouped according to liver function as follows: patients with ACLF and patients without ACLF ("no liver failure", NLF).

\section{Liver cirrhosis and ACLF}

ACLF was defined according to the definition of the Chronic Liver Failure (CLIF) Consortium [3]. Presence of liver cirrhosis was diagnosed based on a combination of characteristic clinical (e.g., ascites, caput medusae, spider angiomata, etc.), laboratory and radiological findings (typical morphological changes of the liver, signs of portal hypertension, etc., in ultrasonography or computed tomography scanning), or via histology, if available [12].

\section{Medication}

All patients received meropenem $1 \mathrm{~g}$ quid $8 \mathrm{~h}$ (Dr. Friedrich Eberth Arzneimittel GmbH, Germany). Meropenem was diluted in $50 \mathrm{~mL}$ isotonic saline solution and given over 30 min by syringe pump via a central venous line (short-term infusion).

\section{Sampling and storage}

We obtained prefilter blood samples at the following time points: T0 as the baseline before the first monitored infusion, $1 \mathrm{~h}$ (T1), $2 \mathrm{~h}$ (T2), $4 \mathrm{~h}$ (T4), $6 \mathrm{~h}$ (T6) and $8 \mathrm{~h}$ after the start of infusion (T8). T8 was obtained before the next infusion of meropenem as a minimum concentration. Furthermore, we obtained values after $24 \mathrm{~h}$ (before and 30 min after end of infusion, T24 and T25) and after 48 h (T48 and T49). All samples were centrifuged immediately, and the supernatant stored at $-20{ }^{\circ} \mathrm{C}$ until assayed.

\section{Assay}

An aliquot of a human serum sample $(250 \mu \mathrm{L})$ was mixed with $50 \mu \mathrm{L}$ of the internal standard ertapenem $(0.2 \mathrm{mg} /$ mL). $500 \mu \mathrm{L}$ acetonitrile was added to precipitate serum proteins. After centrifugation at $14500 \mathrm{rpm}$ for $5 \mathrm{~min}$ at $20{ }^{\circ} \mathrm{C}, 100 \mu \mathrm{L}$ of the supernatant was diluted to a total of $1000 \mu \mathrm{L}$ with water in a glass microvial. The injection volume was $50 \mu \mathrm{L}$.

A validated high-performance liquid chromatography with diode array detection (HPLC-DAD) method was used for the analysis of meropenem serum samples. Chromatography was performed on a Thermo Scientific Accela Liquid chromatography system consisting of an autosampler, quaternary pump and a photo diode array detector with a thermostated column compartment (Thermo Fisher Scientific, Waltham, MA, USA). The chromatographic separation of meropenem and internal standard was carried out on a reversed phase Varian Polaris C18-A $(250 \times 4.0 \mathrm{~mm})$ with particle size of $5 \mu \mathrm{m}$ and a C18-pre-column (SecurityGuard ${ }^{\mathrm{TM}}$ Phenomenex $^{\circledR}$, Aschaffenburg, Germany) using a flow rate of $1.0 \mathrm{~mL} /$ min at $25{ }^{\circ} \mathrm{C}$. The mobile phases consisted of water with $0.2 \%$ ortho-phosphoric acid added (A) and acetonitrile (B). The following gradient program was used (proportion of solution (B): $5 \%$ at $0.0 \mathrm{~min}, 20 \%$ at $6.0 \mathrm{~min}, 70 \%$ at $11.0 \mathrm{~min}$ and $5 \%$ at $14.0 \mathrm{~min}$. Between injections, the sampling needle was flushed with $400 \mu \mathrm{L}$ and washed with $200 \mu \mathrm{L}$ methanol. The wavelength detection was set at $260 \mathrm{~nm}$ and $310 \mathrm{~nm}$. Chromeleon ${ }^{\mathrm{TM}} 7$ Chromatography Data Systems Software (Thermo Fisher Scientific, Waltham, MA, USA) was used for the control of the instruments and data acquisition. The assay was routinely calibrated using six standards of spiked blank human serum $(1,10,20,50,100,150 \mathrm{mg} / \mathrm{L})$ and there were also two independently prepared quality control samples (16 and $64 \mathrm{mg} / \mathrm{L}$ ) included in each analytical series. Validation parameters including accuracy, interferences, linearity of calibration, matrix effects and in-process stability complied with international standards. Regular external quality control is performed by periodical proficiency testing. The limit of quantification was $1 \mathrm{mg} / \mathrm{L}$. 
Continuous veno-venous hemodialysis

CVVHD was performed with Multifiltrate ${ }^{\circledR}$ dialysis machines using an Ultraflux ${ }^{\circledR}$ AV1000S hollow-fiber hemofilter (Fresenius Medical Care, Bad Homburg, Germany) with a membrane surface area of $1.8 \mathrm{~m}^{2}$. Dialyzers and lines were steam sterilized. A regional citrate-calcium anticoagulation was used. No filter change occurred during the study period. The targeted dialysate dose was $30 \mathrm{ml} / \mathrm{kg} / \mathrm{h}$ of actual body weight.

\section{Patient characteristics}

Additional data were obtained from the patients' electronic records (Integrated Care Manager ICM, version 9.1, Drägerwerk, Lübeck, Germany, and Soarian Clinicals 4.01 SP08, Cerner Health Services, Idstein, Germany).

The Acute Physiology and Chronic Health Evaluation II (APACHE II) score [13] and the Sequential Organ Failure Assessment (SOFA) score [14] were recorded on the first day of examination as measures of disease severity. ACLF patients were further characterized by the Model of End-Stage Liver Disease (MELD) score, the Chronic Liver Failure Consortium (CLIF)-SOFA score, and the CLIF-lactate score [12].

\section{Statistics}

Microsoft Excel 2016 (Microsoft Corp., Redmond, WA, USA) was used for data management. The SPSS statistical software package (version 25, IBM Inc., Armonk, NY, USA) was used for descriptive statistical analysis. The pharmacokinetic analysis and Monte Carlo analysis were done with the non-linear mixed-effects modeling software NONMEM, version 7.4 (Icon Development Solutions, Ellicott City, MD, USA). Data are given as median and quartiles.

\section{Pharmacokinetic analysis}

The population PK analysis comprised the development of a structural and statistical base model followed by a categorical evaluation of PK parameter differences between the ACLF and NLF patient population. One and two-compartment PK models with serum data attributed to the central compartment were evaluated. Elimination from the central compartment and intercompartmental distribution were assessed using linear processes via ordinary differential equations. Interindividual variability as described by the coefficient of variation of the exponential model was implemented on clearance, intercompartmental clearance, and peripheral volume of distribution $\left(V_{2}\right)$. Interindividual variability in PK parameters, and different residual unexplained variability models to quantify the difference between the model predictions and observations were investigated. The informativeness of interindividual variability and residual unexplained variability parameters was assessed based on shrinkage [15].
The model quality was evaluated by visual inspection of the observed and predicted meropenem concentrations, residuals and individual predicted PK parameter scatter plots as well as visual predictive checks $(n=1000$ simulations) [16]. Statistical comparisons between nested models with additional covariates were made using the likelihood-ratio test [17]. Because of limited data for the first declining phase of the meropenem concentrationtime profile and large parameter imprecision and parameter correlation between intercompartment clearance and central volume of distribution $\left(V_{1}\right), V_{1}$ was fixed at 8.31 $\mathrm{L}$ as plausible value previously described for critically ill patients [18].

\section{Probability of target attainment (PTA)}

Based on the PK parameters of the final population PK model, Monte Carlo simulations $(n=1000)$ were performed to determine the PTA for different meropenem dosing regimens in ACLF and NLF patients. PTA was defined as time above $4 \times$ the European Committee on Antimicrobial Susceptibility Testing (EUCAST) epidemiological cut-off values (ECOFF) as typical minimum inhibitory concentration (MIC) values $[19,20]$ of the free fraction of meropenem $\left(f T>_{4 \times \mathrm{ECOFF}}\right)$. PTA for achieving a $\mathrm{PK} /$ pharmacodynamic target of $95 \%$ of $f \mathrm{~T}>_{4 \times \mathrm{ECOFF}}$ was calculated on the first day of the therapy and of $100 \%$ $f \mathrm{~T}>_{4 \times \mathrm{ECOFF}}$ at steady-state on day 7 . A dosing regimen was considered adequate if the PTA was $\geq 90 \%$.

PTA was calculated for the following ECOFFs: 0.25 $\mathrm{mg} / \mathrm{L}$ for Enterobacterales and $2.0 \mathrm{mg} / \mathrm{L}$ for Pseudomonas aeruginosa or Acinetobacter baumannii and the following dosing regimens: meropenem $1 \mathrm{~g}$ short-term infusion (over $30 \mathrm{~min}$ ) quid $8 \mathrm{~h}, 1$ g quid $8 \mathrm{~h}$ as prolonged infusion over $4 \mathrm{~h}$ after a short-term infusion loading dose of $2 \mathrm{~g}$ and without loading dose, $2 \mathrm{~g}$ short-term infusion quid 8 $\mathrm{h}$, and continuous infusion of meropenem $3 \mathrm{~g} /$ day after a short-term infusion loading dose of $2 \mathrm{~g}$.

\section{Results}

A total of 19 critically ill patients were included in this study with eight patients suffering from ACLF and renal failure and 11 patients only from renal failure. ACLF patients had a mean MELD score of 34 (27-37), a CLIFSOFA of 15 (14-15) and a CLIF-lactate score of 59 (5764). An overview of the patients' characteristics is given in Table 1. Two ACLF patients were admitted for variceal hemorrhage, two for pneumonia, two for spontaneous bacterial peritonitis, one for urosepsis, and one for hepatorenal syndrome. Gram-positive bacteria were identified in four patients in microbiological sampling, Escherichia coli and Candida spp. in one case each, and no pathogens were found in two patients. In the NLF group, five patients were treated for hospital acquired pneumonia, 
Table 1 Patients' characteristics

\begin{tabular}{|c|c|c|c|}
\hline Parameter & ACLF & NLF & $p$ \\
\hline Number of patients & $n=8$ & $n=11$ & \\
\hline Age [years] & $59(46-68)$ & $61(55-75)$ & 0.31 \\
\hline \multirow[t]{2}{*}{ Gender } & Males: 6 & Males: 8 & 1.0 \\
\hline & Females: 2 & Females: 3 & \\
\hline Weight [kg] & $78(60-81)$ & $77(55-86)$ & 0.97 \\
\hline Height $[\mathrm{cm}]$ & $175(166-180)$ & 175 (170-184) & 0.49 \\
\hline APACHE $\|$ & $30(25-40)$ & $32(20-38)$ & 0.97 \\
\hline SOFA & $16(15-19)$ & $14(11-19)$ & 0.44 \\
\hline Blood flow rate $\left[\mathrm{mL}^{*} \mathrm{~h}^{-1}\right]$ & $100(100-200)$ & $100(100-200)$ & 0.84 \\
\hline Dialysate rate $\left[\mathrm{mL}^{*} \mathrm{~h}^{-1}\right]$ & $2000(2000-2750)$ & 2000 (2000-2000) & 1.0 \\
\hline Ultrafiltrate $\left[\mathrm{mL}^{*} \mathrm{~h}^{-1}\right]$ & $25(0-138)$ & $150(50-200)$ & 0.17 \\
\hline $\begin{array}{l}\text { Dialysate dose } \\
\qquad\left[\mathrm{ml}^{*} \mathrm{~kg}^{-1 *} \mathrm{~h}^{-1}\right]\end{array}$ & $31(23-37)$ & $26(23-40)$ & 0.90 \\
\hline $\begin{array}{l}\text { Patients receiving } \\
\text { norepinephrine } \geq 0.01 \\
\mu \mathrm{g}^{*} \mathrm{~kg}^{-1 *} \mathrm{~min}^{-1}\end{array}$ & $88 \%$ & $73 \%$ & 0.60 \\
\hline PT [\%] & $50(29-61)$ & $87(74-103)$ & 0.001 \\
\hline Bilirubin [mg/dL] & $7.4(4.3-18.7)$ & $2.1(0.4-8.3)$ & 0.05 \\
\hline Antithrombin [\%] & $48(22-54)$ & $71(56-90)$ & 0.004 \\
\hline
\end{tabular}

$A C L F$ acute-on-chronic liver failure due to liver cirrhosis, NLF patients without liver failure, APACHE II Acute Physiology and Chronic Health Evaluation, SOFA Sequential Organ Failure Assessment Score, $P T$ prothrombin time, ns not statistically significant, data are given as median and quartiles

four patients for peritonitis and one patient each for cholecystitis and for complicated soft tissue infection. Microbiological sampling detected two cases of Pseudomonas aeruginosa, Enterobacter cloacae, Klebsiella pneumoniae, Serratia marcescens, and yeasts in one case each, Grampositive bacteria in four patients, and was without results in three patients. Seven patients (88\%) suffering from ACLF and eight (73\%) patients in the NLF group died during the intensive care stay $(p=0.60)$. Ascites was present in $75 \%$ of ACLF patients.

\section{Meropenem serum concentrations and pharmacokinetic model}

In total, 19 meropenem measurements were available per sampling time point for the first study dosing interval on day 1 and day 2 with values missing for T4 to T8 for 2 patients and 17 measurements per sampling time point available on day $3\left(n_{\text {total }}=180\right)$. The meropenem clearance of the CVVHD was $4.99(4.32-5.75) \mathrm{L} / \mathrm{h}$.

Meropenem PK in patients with and without ACLF was described by a two-compartment PK model with linear clearance from the central compartment. Interindividual variability for clearance was coefficient of variation $=28 \%$ and on $V_{2}=66 \%$. Separate estimation of $V_{2}$ yielded a $V_{2}$ of $18.5 \mathrm{~L}$ for NLF patients and $35.5 \mathrm{~L}$ for ACLF patients $(p=0.05$, likelihood-ratio testing), which largely explained interindividual variability in $V_{2}(61.9 \%$
Table 2 Parameter estimates for meropenem from the final covariate two-compartment population pharmacokinetic model

\begin{tabular}{llll}
\hline Parameter & ACLF & NLF & $\mathbf{p}$ \\
\hline Structural pharmacokinetic parameters for meropenem (RSE, \%) & \\
$\mathrm{CL}[\mathrm{L} / \mathrm{h}]$ & $5.06(6.70)$ & & $\mathrm{ns}$ \\
$V_{1}[\mathrm{~L}]$ & $8.31^{\mathrm{a}}$ & & $\mathrm{n} / \mathrm{a}$ \\
$V_{2}[\mathrm{~L}]$ & $35.5(33.3)$ & $18.5(33.2)$ & 0.05 \\
$\mathrm{Q}[\mathrm{L} / \mathrm{h}]$ & $7.23(9.80)$ & & $\mathrm{n} / \mathrm{a}$
\end{tabular}

Interindividual variability parameters for meropenem (RSE, \%)

$\mathrm{CL}, \mathrm{CV}[\%] \quad 29.8(16.8) \quad \mathrm{n} / \mathrm{a}$

Q, CV [\%] $27.6(28.8) \quad n / a$

Residual variability parameter $22.0(8.40) \quad \mathrm{n} / \mathrm{a}$ o proportional, CV \%

RSE is presented on the approximated standard deviation scale

$A C L F$ acute-on-chronic liver failure, NLF patients without liver failure, $C L$ clearance, $V_{1 / 2}$ central/peripheral volume of distribution, $Q$ intercompartmental clearance, CV coefficient of variation, $n s$ not statistically significant, $n / a$ not applicable, $R S E$ relative standard error

a Value fixed (see "Methods" section)

relative reduction). The remaining interindividual variability in $V_{2}$ was estimated imprecisely and suffered from a large shrinkage (50.8\%), indicating non-informativeness of this parameter by the data. Due to the model stability, it was excluded in the final model. No difference in clearance between the two patient populations was evident (ACLF: $5.20 \mathrm{~L} / \mathrm{h}, \mathrm{NLF}: 5.11 \mathrm{~L} / \mathrm{h} ; p=0.90)$. The halflives of ACLF compared to NLF patients were $t_{1 / 2 \alpha}: 0.43$ vs. $0.40 \mathrm{~h}(p=0.60)$ and $t_{1 / 2}: 9.0 \mathrm{vs.} 5.0 \mathrm{~h}(p=0.002)$. The final model parameter values are depicted in Table 2.

\section{Target attainment}

PTA for the lower ECOFF of $0.25 \mathrm{mg} / \mathrm{L}$ (Enterobacterales) was $100 \%$ for all dosing regimens on day 1 (Fig. 1) and on day 7 (Fig. 2) in both groups. For the higher ECOFF of $2.0 \mathrm{mg} / \mathrm{L}$ (P. aeruginosa, A. baumannii), meropenem $1 \mathrm{~g}$ short-term infusion quid $8 \mathrm{~h}$ yielded a PTA of $18 \%$ in the ACLF group and $48 \%$ in the NLF group on day 1 and $80 \%$ for ACLF and 65\% for NLF patients on day 7 . With prolonged infusion of $1 \mathrm{~g}$ quid $8 \mathrm{~h}$, PTA was increased to $30 \%$ and $67 \%$ on day 1 and $88 \%$ and $81 \%$ on day 7 for ACLF and NLF patients, respectively. For a prolonged infusion after a short-term infusion loading dose of $2 \mathrm{~g}$, PTA was higher with $94 \%$ and $91 \%$ on day 1 and $88 \%$ and $83 \%$ on day 7 . The dosing regimen of $2 \mathrm{~g}$ shortterm infusion quid $8 \mathrm{~h}$ yielded a PTA of $85 \%$ and $91 \%$ on day 1 and $98 \%$ and $93 \%$ on day 7 . The continuous infusion of $3 \mathrm{~g}$ quid $8 \mathrm{~h}$ after a short-term infusion loading dose of $2 \mathrm{~g}$ reached a PTA of $100 \%$ for both groups on days 1 and 7 . 

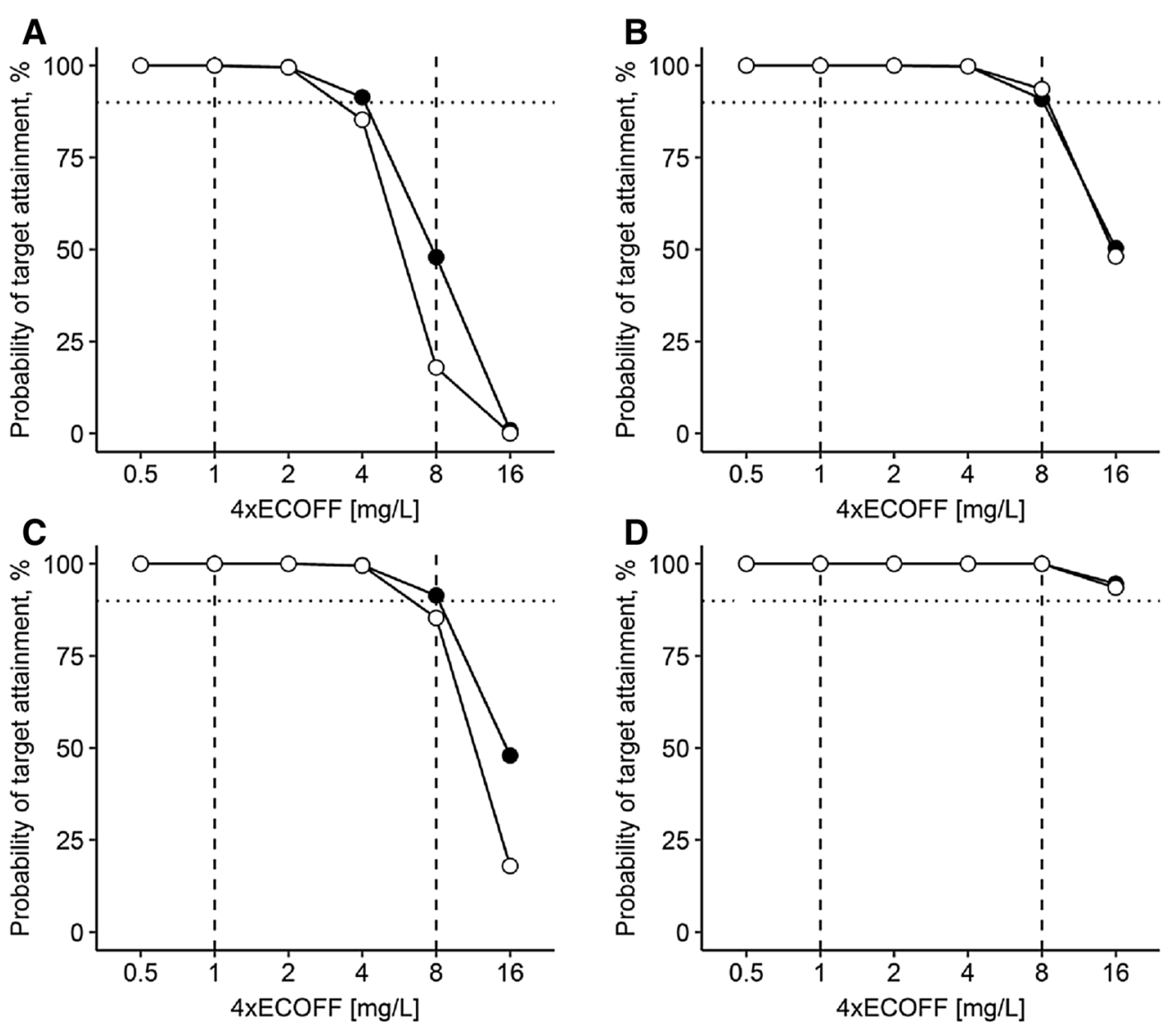

Fig. 1 Probability of target attainment on day 1. a $1 \mathrm{~g}$ quid $8 \mathrm{~h}$ (30 min); b $2 \mathrm{~g}$ loading dose (30 min) followed by $1 \mathrm{~g}$ prolonged infusion (over 4 h) quid 8 h; $\mathbf{c} 2 \mathrm{~g}$ quid $8 \mathrm{~h}$ (30 min); d $2 \mathrm{~g}$ loading dose (30 min) followed by continuous infusion of $3 \mathrm{~g} /$ day. Empty dots: patients with acute-on-chronic liver failure, closed dots: patients without liver failure, dotted horizontal line depicts probability of target attainment $\geq 90 \%$, dashed vertical lines depict typical target concentrations as 4x European Committee on Antimicrobial Susceptibility Testing epidemiological cut-off

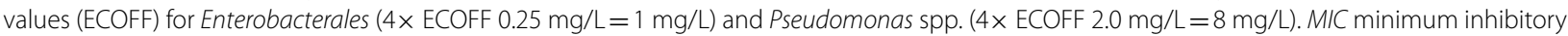
concentration

Therefore, adequate PTA was achieved for all dosing regimens up to an ECOFF of $0.25 \mathrm{mg} / \mathrm{L}$. However, for the higher ECOFF of $2 \mathrm{mg} / \mathrm{L}$, only a $2 \mathrm{~g}$ short-term infusion followed by a continuous infusion of $3 \mathrm{~g} /$ day reached adequate PTA on day 1 and 7 in both groups. A dosing regimen of $2 \mathrm{~g}$ quid $8 \mathrm{~h}$ was sufficient on day 1 only in the NLF group and in both groups at steady-state.

\section{Discussion}

In this study, we assessed the impact of ACLF on PK and PTA of meropenem in critically ill patients undergoing CVVHD. For the ECOFF values typical for Enterobacterales, all simulated dosing regimens yielded an adequate PTA. However, for non-fermenting bacteria with higher ECOFF values like Pseudomonas spp. or Acinetobacter spp., the standard dosing regimen of $1 \mathrm{~g}$ quid $8 \mathrm{~h}$ failed to achieve adequate PTA at start and steady-state in ACLF. In contrast, dosing regimens of $2 \mathrm{~g}$ short-term infusion quid $8 \mathrm{~h}$ or the continuous dosing regimen of $3 \mathrm{~g} /$ day showed adequate PTA at steady-state. Additionally, the larger $V_{2}$ in ACLF patients required higher loading doses to achieve similar PTA compared to NLF patients during day 1.

Meropenem is often used as empiric broad-spectrum antimicrobial therapy in patients with ACLF suffering from sepsis and septic shock. These critically ill patients often suffer from multiple organ failure and receive renal replacement therapy. The Food and Drug Administration (FDA) label states that insufficient data are available for an informed adjustment of the dosing regimen of meropenem on renal replacement therapy, but several small studies have evaluated the PK on different types of renal replacement therapy by now [21-24]. According to both FDA and European Medicines Agency (EMA) labeling, no adjustment of the dosing regimen is required in patients with hepatic impairment. However, 


\section{A}
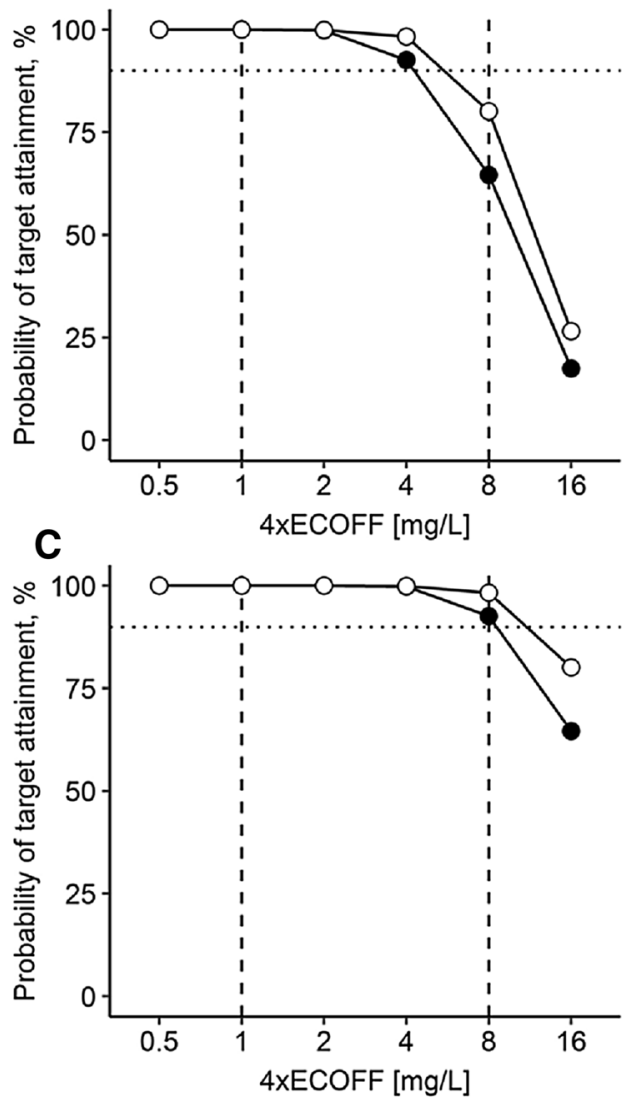

B
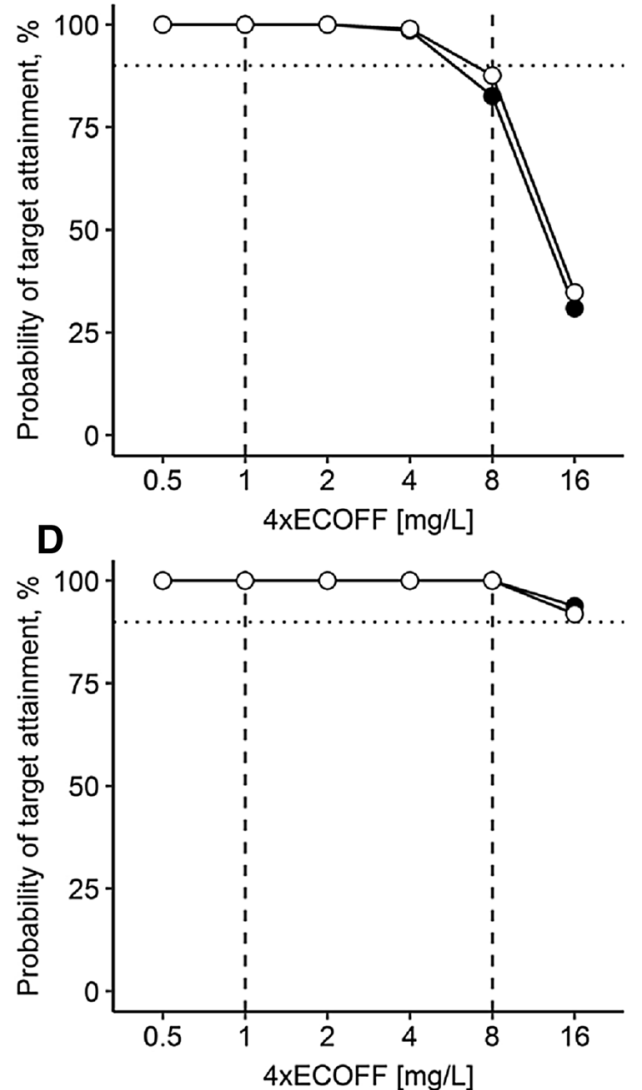

Fig. 2 Probability of target attainment at steady-state (day 7). a 1 g quid 8 h (30 min); b 2 g loading dose (30 min) followed by $1 \mathrm{~g}$ prolonged infusion (over 4 h) quid 8 h; c 2 g quid 8 h (30 min); d 2 g loading dose (30 min) followed by continuous infusion of 3 g/day. Empty dots: patients with acute-on-chronic liver failure, closed dots: patients without liver failure, dotted horizontal line depicts probability of target attainment $\geq 90 \%$, dashed vertical lines depict typical target concentrations as 4x European Committee on Antimicrobial Susceptibility Testing epidemiological cut-off

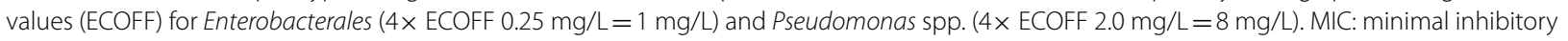
concentration

these recommendations are based on data obtained in non-critically ill patients with single organ failure and do not take into account the complex changes of distribution and elimination kinetics in ACLF [11]. Furthermore, antibiotic therapy must "hit hard" especially in the initial phase to prevent an increase in sepsis-related mortality, foremost in ACLF patients [25] and insufficient antibiotic concentrations have been linked to an increase in mortality, particularly in critically ill patients [26].

In a recent meta-analysis, a decrease in mortality has been shown for prolonged or continuous meropenem infusions that achieve concentrations for a longer time above the MIC as compared to bolus applications [27] and has been recently recommended in critically ill patients [28]. Therapeutic drug monitoring has been proposed to detect insufficient antibiotic concentrations and to allow for adjustments in the dosing regimen, but therapeutic drug monitoring is not ubiquitously available and often does not allow for immediate adjustments after the initiation of antibiotic therapy. Therefore, PK models predicting drug distribution and elimination kinetics are useful in the critical care setting until therapeutic drug monitoring results allowing for targeted adjustments are available.

Currently, there are no PK data available in patients with ACLF. It has been shown for other beta-lactam antibiotics that $\mathrm{V}$ increases in patients with liver cirrhosis and ascites $[29,30]$ and that meropenem is readily redistributed into the peritoneal fluid in septic shock [31]. Hence, our finding of a nearly twice as high $V_{2}$ for ACLF compared to NLF patients is plausible. As ascites was present in most ACLF patients, we assume that the ascites is the most important factor in the increase in $V_{2}$. All patients in the NLF group as controls were admitted for sepsis or septic shock and it is known that $V_{2}$ is already increased in these conditions [32]. Therefore, in 
ACLF patients $V_{2}$ was even increased further above the increase in sepsis or septic shock.

No difference in the clearance of meropenem was found between the two groups. It has been shown before that 80 to $90 \%$ of meropenem and its metabolites are renally eliminated [23], that the clearance mainly depends on renal clearance $[33,34]$, and that liver impairment does not influence the clearance [11]. In our study cohort with critically ill patients, no difference in clearance could be shown either, confirming the previous results. Notably, in our patient population, the clearance of approximately 5 $\mathrm{L} / \mathrm{h}$ was lower than 8.3 to $9.3 \mathrm{~L} / \mathrm{h}$ as previously reported $[35,36]$, but comparable to a clearance of $4.8 \mathrm{~L} / \mathrm{h}$ as shown for anuric patients receiving renal replacement therapy [37]. Therefore, the meropenem elimination in our cohort most likely relied solely on the renal replacement therapy in both groups. All other parameter values were comparable to those previously reported for critically ill patients [36]. A multitude of clinical studies of meropenem PK in healthy volunteers and critically ill patients, based on extensive sampling data, described meropenem concentration-time profiles as bi-exponential owing to the vast distribution in blood and into various tissues [38-40]. Hence, in agreement with previous analyses of meropenem PK, in our study a two-compartment model with linear clearance from the central compartment was employed to describe meropenem PK.

We defined target attainment as reaching a minimum concentration four times above the respective ECOFF values for $100 \%$ of the time at steady-state (day 7) and of $95 \%$ on day 1 to account for meropenem concentrations below $4 \times$ ECOFF during the initial increase at the beginning of the first infusion without negatively affecting target attainment. Although lower target values of $50 \%$ or $100 \%$ of the time above a single MIC, or $40 \%, 50 \%$, and $70 \%$ above the fourfold MIC have been proposed [26, 41], several studies and a recent guideline support our choice of $100 \% f \mathrm{~T}>_{4 \times \mathrm{ECOFF}}$ [28]: an optimized outcome could be shown for meropenem concentrations continuously exceeding 4.3-fold MIC for other beta-lactam antibiotics [42] and in vitro studies with Pseudomonas aeruginosa even determined the maximum activity of ceftazidime at concentrations of the 6.6-fold MIC [43]. No additional killing was shown when increasing the concentrations from 5- to 20-fold MIC [44]. This strengthens the recommendation for continuous attainment of four to eightfold MIC for adequate PTA [28].

Antibiotic concentrations are usually measured in serum and do not reflect the concentrations at the infectious focus that should be above the MIC to allow for a continuous bactericidal effect. Meropenem's penetration for peritoneal fluid is of approximately $70 \%$ [31], but only $25 \%$ for lung epithelial lining fluid and lung tissue [45, 46] and 20\% for cerebrospinal fluid [47]. In cases of low penetration, it has been doubted that sufficient concentrations are attained for a sustained bactericidal effect [45]. This may explain why higher concentrations than the MIC are required for a continuous bactericidal effect and improved outcome.

On the other hand, neurotoxicity is a known side effect of meropenem when high concentrations are maintained continuously. Steady-state minimum concentrations above $64 \mathrm{mg} / \mathrm{L}$ were shown to be associated with a $50 \%$ chance of developing neurotoxicity [48]. Our deterministic simulations suggested that our selected dosing regimens were safe as the trough concentrations were well below this threshold in the typical ACLF and NLF patient (Fig. 3).

It has been proposed that higher targets above the MIC also reduce the development of antibiotic resistance [49], but this concept has been challenged [50, 51] and further data are required.

With bolus application of beta-lactams, high peak concentrations are attained, but trough concentrations are often below the MIC or the targeted multiple of the MIC so that no continuous bactericidal effect is achieved. Patients' outcome may improve with a longer time above the MIC $[26,52]$. In patients with ACLF and a meropenem total dose of $3 \mathrm{~g} /$ day, PTA increased with a slower application of the respective $1 \mathrm{~g}$ aliquots, reaching $100 \%$ for the continuous infusion (Fig. 2).

Several attempts have been made to develop dosing nomograms and risk assessments for target non-attainment for meropenem in critically ill patients [33, 34, $36,53,54]$, but all approaches have mainly focused on the clearance at steady-state due to the primarily renal elimination. Although ACLF does not significantly alter clearance, the loading dose of meropenem needs special attention due to the increased V in ACLF patients. Our data strongly suggest administering a loading dose of $2 \mathrm{~g}$ meropenem in ACLF especially if $P$. aeruginosa or other non-fermenting bacteria with a high ECOFF or MIC are considered as causative organisms and to continue the therapy by continuous infusion to achieve concentrations above $4 \times$ ECOFF throughout the therapy. As soon as the steady-state is reached, no further adjustment of the meropenem dose is necessary in ACLF patients, as the PK parameters differ only insignificantly between the groups.

Our study has certain limitations. First, the number of patients was rather small and this necessarily limits the precision of the PK parameters. However, this is a common number of patients for pharmacokinetic studies and the first study assessing PK data in ACLF.

Second, we have decided to select high target concentrations with the use of the fourfold ECOFF as the basis 

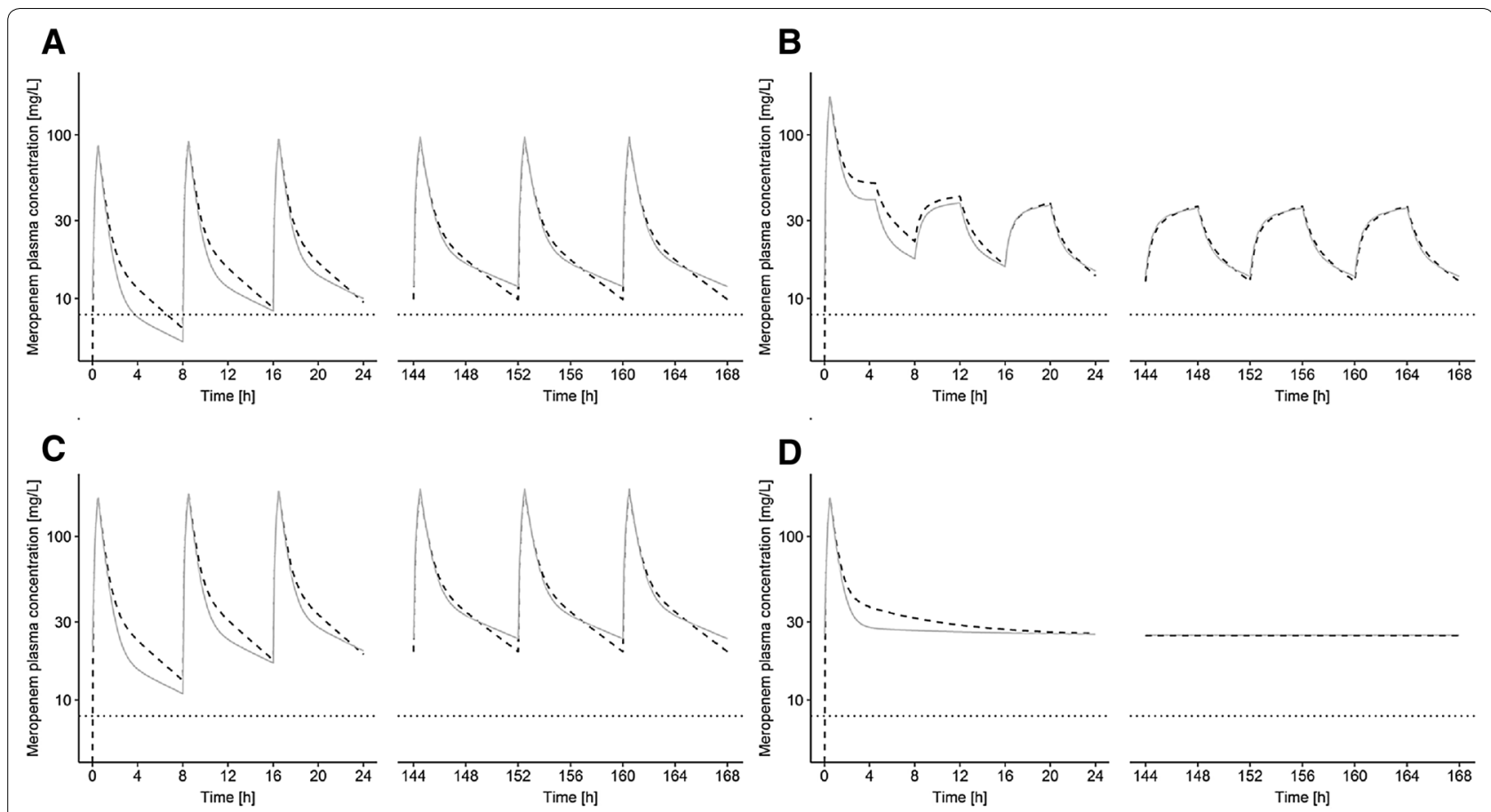

Fig. 3 Deterministic simulations of meropenem. a $1 \mathrm{~g}$ quid $8 \mathrm{~h}$ (30 min); b $2 \mathrm{~g}$ loading dose (30 min) followed by $1 \mathrm{~g}$ prolonged infusion (over $4 \mathrm{~h}$ ) quid 8 h; c $2 \mathrm{~g}$ quid $8 \mathrm{~h}$ (30 min); $\mathbf{d} 2 \mathrm{~g}$ loading dose (30 min) followed by continuous infusion of $3 \mathrm{~g} /$ day. Solid lines: typical patient without liver failure; dashed line: typical acute-on-chronic liver failure patient; dotted horizontal line depict typical target concentrations as $4 x$ European Committee on Antimicrobial Susceptibility Testing epidemiological cut-off values (ECOFF) for Pseudomonas spp. (4× ECOFF $2.0 \mathrm{mg} / \mathrm{L}=8 \mathrm{mg} / \mathrm{L}$ )

for PTA calculations, but these concentrations are recommended by guidelines [28].

Third, we did not measure the free fraction of meropenem. However, meropenem is only negligible bound to albumin at $2 \%$ [38].

\section{Conclusion}

We evaluated the PTA of meropenem in critically ill patients with ACLF undergoing CVVHD for the fourfold ECOFF values for Enterobacterales and Pseudomonas spp. For Enterobacterales, all simulated dosing regimens including the standard $1 \mathrm{~g}$ short-term infusion quid 8 $\mathrm{h}$ dosing regimen yielded a PTA of $100 \%$ and therefore resulted in adequate drug exposure. For Pseudomonas spp. only the dosing regimens of $2 \mathrm{~g}$ short-term infusion quid $8 \mathrm{~h}$ and the continuous dosing regimen of $3 \mathrm{~g} /$ day showed adequate PTA at steady-state. Compared to NLF patients who were suffering from sepsis or septic shock with an already increased $V_{2}$ as compared to non-critically ill patients, ACLF patients showed an even higher $V_{2}$ highlighting the importance of sufficient loading doses to attain adequate PTA on the first day of therapy. No dose adjustments in ACLF patients were necessary at steady-state. However, over time the course of antibiotic therapy, therapeutic drug monitoring of meropenem might aid in the guidance of optimal antibiotic treatment.

\begin{abstract}
Abbreviations
ACLF: Acute-on-chronic liver failure; CLIF: Chronic Liver Failure Consortium; CVVHD: Continuous veno-venous hemodialysis; ECOFF: European Committee on Antimicrobial Susceptibility Testing epidemiological cut-off values; EUCAST: European Committee on Antimicrobial Susceptibility Testing; $f T>_{4 \times E C O F F}$ :Time of the free antibiotic fraction above the fourfold of the European Committee on Antimicrobial Susceptibility Testing epidemiological cut-off value; MIC: Minimal inhibitory concentration; MELD: Model for end-stage liver disease; NLF: No liver failure; n.s.: Not statistically significant; PK: Pharmacokinetics; PTA: Probability of target attainment; SOFA: Sequential Organ Failure Assessment; $V$ : Volume of distribution; $V_{1}$ : Central volume of distribution; $V_{2}$ : Peripheral volume of distribution.
\end{abstract}

\section{Acknowledgements}

We are indebted to our study nurses and the teams of the participating intensive care units for their valuable help in the data acquisition.

\section{Authors' contributions}

$J G$ designed the study, wrote the manuscript and helped to acquire and interpret the data. DB performed the pharmacokinetic analysis and the Monte Carlo analysis and helped to write the manuscript. CKö helped to design the study and to write the manuscript. KR helped with the data management and interpretation. WJ performed part of the pharmacokinetic analysis. DJ acquired the data. SIB developed the analytical method and analyzed the samples. CM helped to interpret the data and to write the manuscript. SK helped with study design and to acquire funding. CKI helped with the pharmacokinetic analysis and the Monte Carlo analysis, to interpret the data 
and to write the manuscript. VF designed the study, acquired funding and interpreted the data. All authors read and approved the final manuscript.

\section{Funding}

This study was supported in part by the DAMP-Stiftung, Kiel, Germany, Reference No. 2017-20. Further expenses were financed by departmental funds.

\section{Availability of data and materials}

Data are available from the authors upon reasonable request.

\section{Ethics approval and consent to participate}

The study was approved by the Ethics Committee of the Hamburg Chamber of Physicians, Germany (Reference: PV5415). Consent was obtained from the patients' closest relatives or legal surrogates.

\section{Consent for publication}

Not applicable.

\section{Competing interest}

All authors certify that they have no conflicts of interests.

\section{Author details}

${ }^{1}$ Department of Intensive Care Medicine, University Medical Center HamburgEppendorf, Martinistraße 52, 20246 Hamburg, Germany. ${ }^{2}$ Department of Clinical Pharmacy and Biochemistry, Institute of Pharmacy, Freie Universitaet Berlin, Kelchstraße 31, 12169 Berlin, Germany. ${ }^{3}$ Graduate Research Training Program PharMetrX, Berlin, Germany. ${ }^{4}$ Hospital Pharmacy, University Medical Center Hamburg-Eppendorf, Martinistraße 52, 20246 Hamburg, Germany. ${ }^{5}$ Department of Pharmaceutical Chemistry, University of Vienna, Althanstraße 14, 1090 Vienna, Austria. ${ }^{6}$ Department of Legal Medicine, University Medical Center Hamburg-Eppendorf, Butenfeld 34, 22529 Hamburg, Germany. ${ }^{7}$ First Department of Internal Medicine and Gastroenterology, University Medical Center Hamburg-Eppendorf, Martinistraße 52, 20246 Hamburg, Germany.

${ }^{8}$ Department of Medicine B, Münster University Hospital, Albert-Schweitzer-Campus 1, 48149 Münster, Germany.

\section{Received: 25 October 2019 Accepted: 13 April 2020}

Published online: 22 April 2020

\section{References}

1. Fernandez J, Acevedo J, Wiest R, Gustot T, Amoros A, Deulofeu C, Reverter E, Martinez J, Saliba F, Jalan R, et al. Bacterial and fungal infections in acute-on-chronic liver failure: prevalence, characteristics and impact on prognosis. Gut. 2018;67(10):1870-80.

2. Hubener P, Braun G, Fuhrmann V. Acute-on-chronic liver failure: a diagnostic and therapeutic challenge for intensive care. Med Klin Intensivmed Notfmed. 2018;113(8):649-57.

3. Moreau R, Jalan R, Gines P, Pavesi M, Angeli P, Cordoba J, Durand F, Gustot T, Saliba F, Domenicali M, et al. Acute-on-chronic liver failure is a distinct syndrome that develops in patients with acute decompensation of cirrhosis. Gastroenterology. 2013;144(7):e1426-37.

4. European Association for the Study of the Liver. EASL clinical practice guidelines for the management of patients with decompensated cirrhosis. J Hepatol. 2018;69(2):406-60.

5. Fuhrmann $V$, Whitehouse T, Wendon J. The ten tips to manage critically ill patients with acute-on-chronic liver failure. Intensive Care Med. 2018:44(11):1932-5.

6. Jalan R, Fernandez J, Wiest R, Schnabl B, Moreau R, Angeli P, Stadlbauer V, Gustot T, Bernardi M, Canton R, et al. Bacterial infections in cirrhosis: a position statement based on the EASL special conference 2013. J Hepatol. 2014;60(6):1310-24.

7. Fernandez J, Prado V, Trebicka J, Amoros A, Gustot T, Wiest R, Deulofeu C, Garcia E, Acevedo J, Fuhrmann V, et al. Multidrug-resistant bacterial infections in patients with decompensated cirrhosis and with acute-onchronic liver failure in Europe. J Hepatol. 2019;70(3):398-411.

8. Rhodes A, Evans LE, Alhazzani W, Levy MM, Antonelli M, Ferrer R, Kumar A, Sevransky JE, Sprung CL, Nunnally ME, et al. Surviving sepsis campaign: international guidelines for management of sepsis and septic shock: 2016. Intensive Care Med. 2017;43(3):304-77.
9. Staufer K, Roedl K, Kivaranovic D, Drolz A, Horvatits T, Rasoul-Rockenschaub S, Zauner C, Trauner M, Fuhrmann V. Renal replacement therapy in critically ill liver cirrhotic patients-outcome and clinical implications. Liver Int. 2017;37(6):843-50.

10. Heintz BH, Matzke GR, Dager WE. Antimicrobial dosing concepts and recommendations for critically ill adult patients receiving continuous renal replacement therapy or intermittent hemodialysis. Pharmacotherapy. 2009;29(5):562-77.

11. Thyrum PT, Yeh C, Birmingham B, Lasseter K. Pharmacokinetics of meropenem in patients with liver disease. Clin Infect Dis. 1997;24(Suppl 2):S184-90.

12. Drolz A, Horvatits T, Rutter K, Landahl F, Roedl K, Meersseman P, Wilmer A, Kluwe J, Lohse AW, Kluge S, et al. Lactate improves prediction of shortterm mortality in critically III patients with cirrhosis: a multinational study. Hepatology. 2019;69(1):258-69.

13. Knaus WA, Draper EA, Wagner DP, Zimmerman JE. APACHE II: a severity of disease classification system. Crit Care Med. 1985;13(10):818-29.

14. Vincent JL, Moreno R, Takala J, Willatts S, De Mendonca A, Bruining H, Reinhart CK, Suter PM, Thijs LG. The SOFA (Sepsis-related Organ Failure Assessment) score to describe organ dysfunction/failure. On behalf of the working group on sepsis-related problems of the European society of intensive care medicine. Intensive Care Med. 1996;22(7):707-10.

15. Savic RM, Karlsson MO. Importance of shrinkage in empirical Bayes estimates for diagnostics: problems and solutions. AAPS J. 2009;11(3):558-69.

16. Holford N. The visual predictive check-superiority to standard diagnostic (Rorschach) plots. In: Populations approach group Europe conference abstract (PAGE 14) 2005.

17. Ette El, Sun H, Ludden TM. Balanced designs in longitudinal population pharmacokinetic studies. J Clin Pharmacol. 1998;38(5):417-23.

18. Ehmann L, Zoller M, Minichmayr I, Scharf C, Frey L, Vogeser M, Huisinga W, Zander J, Kloft C. Pharmacokinetics of meropenem in critically ill patients with varying renal function. In: Populations Approach Group Europe Conference Abstract (PAGE 25) 2016.

19. Antimicrobial wild type distributions of microorganisms https://mic. eucast.org/Eucast2/.

20. Mouton JW, Muller AE, Canton R, Giske CG, Kahlmeter G, Turnidge J. MIC-based dose adjustment: facts and fables. J Antimicrob Chemother. 2017;73(3):564-8.

21. Braune S, Konig C, Roberts JA, Nierhaus A, Steinmetz O, Baehr M, Kluge S, Langebrake C. Pharmacokinetics of meropenem in septic patients on sustained low-efficiency dialysis: a population pharmacokinetic study. Crit Care. 2018;22(1):25.

22. Christensson BA, Nilsson-Ehle I, Hutchison M, Haworth SJ, Oqvist B, Norrby SR. Pharmacokinetics of meropenem in subjects with various degrees of renal impairment. Antimicrob Agents Chemother. 1992;36(7):1532-7.

23. Thalhammer F, Horl WH. Pharmacokinetics of meropenem in patients with renal failure and patients receiving renal replacement therapy. Clin Pharmacokinet. 2000;39(4):271-9.

24. Jamal JA, Mat-Nor MB, Mohamad-Nor FS, Udy AA, Wallis SC, Lipman $J$, Roberts JA. Pharmacokinetics of meropenem in critically ill patients receiving continuous venovenous haemofiltration: a randomised controlled trial of continuous infusion versus intermittent bolus administration. Int J Antimicrob Agents. 2015;45(1):41-5.

25. Choudhury A, Kumar M, Sharma BC, Maiwall R, Pamecha V, Moreau R, Chawla YK, Duseja A, Mahtab M, Rahman S, et al. Systemic inflammatory response syndrome in acute-on-chronic liver failure: relevance of 'golden window': a prospective study. J Gastroenterol Hepatol. 2017;32(12):1989-97.

26. Roberts JA, Paul SK, Akova M, Bassetti M, De Waele JJ, Dimopoulos G, Kaukonen KM, Koulenti D, Martin C, Montravers P, et al. DALI: defining antibiotic levels in intensive care unit patients: are current beta-lactam antibiotic doses sufficient for critically ill patients? Clin Infect Dis. 2014;58(8):1072-83.

27. Yu Z, Pang X, Wu X, Shan C, Jiang S. Clinical outcomes of prolonged infusion (extended infusion or continuous infusion) versus intermittent bolus of meropenem in severe infection: a meta-analysis. PLoS ONE. 2018;13(7):e0201667.

28. Guilhaumou R, Benaboud S, Bennis Y, Dahyot-Fizelier C, Dailly E, Gandia P, Goutelle S, Lefeuvre S, Mongardon N, Roger C, et al. Optimization of the treatment with beta-lactam antibiotics in critically ill patients-guidelines 
from the French society of pharmacology and therapeutics (Societe Francaise de Pharmacologie et Therapeutique-SFPT) and the French Society of Anaesthesia and intensive care medicine (Societe Francaise d'Anesthesie et Reanimation-SFAR). Crit Care. 2019;23(1):104.

29. el Touny M, el Guinaidy M, Abdel Barry M, Osman L, Sabbour MS. Pharmacokinetics of aztreonam in patients with liver cirrhosis and ascites. J Antimicrob Chemother. 1992;30(3):387-95.

30. el Touny M, el Guinaidy MA, el Abd Barry M, Osman L, Sabbour MS. Pharmacokinetics of ceftazidime in patients with liver cirrhosis and ascites. J Antimicrob Chemother. 1991;28(1):95-100.

31. Karjagin J, Lefeuvre S, Oselin K, Kipper K, Marchand S, Tikkerberi A, Starkopf J, Couet W, Sawchuk RJ. Pharmacokinetics of meropenem determined by microdialysis in the peritoneal fluid of patients with severe peritonitis associated with septic shock. Clin Pharmacol Ther 2008;83(3):452-9.

32. Ulldemolins M, Vaquer S, Llaurado-Serra M, Pontes C, Calvo G, Soy D, Martin-Loeches I. Beta-lactam dosing in critically ill patients with septic shock and continuous renal replacement therapy. Crit Care. 2014;18(3):227.

33. Minichmayr IK, Roberts JA, Frey OR, Roehr AC, Kloft C, Brinkmann A. Development of a dosing nomogram for continuous-infusion meropenem in critically ill patients based on a validated population pharmacokinetic model. J Antimicrob Chemother. 2018;73(5):1330-9.

34. Pai MP, Cojutti P, Pea F. Pharmacokinetics and pharmacodynamics of continuous infusion meropenem in overweight, obese, and morbidly obese patients with stable and unstable kidney function: a step toward dose optimization for the treatment of severe gram-negative bacterial infections. Clin Pharmacokinet. 2015;54(9):933-41.

35. Ehmann L, Zoller M, Scharf C, Minichmayr IK, Vogeser M, Frey L, Zander J, Kloft C. Pharmacokinetic/pharmacodynamic analysis of meropenem in critically ill patients. In: ECCMID 2016.

36. Ehmann L, Zoller M, Minichmayr IK, Scharf C, Huisinga W, Zander J, Kloft C. Development of a dosing algorithm for meropenem in critically ill patients based on a population pharmacokinetic/pharmacodynamic analysis. Int J Antimicrob Agents. 2019;54(3):309-17.

37. Burger R, Guidi M, Calpini V, Lamoth F, Decosterd L, Robatel C, Buclin T, Csajka C, Marchetti O. Effect of renal clearance and continuous renal replacement therapy on appropriateness of recommended meropenem dosing regimens in critically ill patients with susceptible life-threatening infections. J Antimicrob Chemother. 2018;73(12):3413-22.

38. Craig WA. The pharmacology of meropenem, a new carbapenem antibiotic. Clin Infect Dis. 1997;24(Suppl 2):S266-75.

39. Ververs TF, van Dijk A, Vinks SA, Blankestijn PJ, Savelkoul JF, Meulenbelt J, Boereboom FT. Pharmacokinetics and dosing regimen of meropenem in critically ill patients receiving continuous venovenous hemofiltration. Crit Care Med. 2000;28(10):3412-6

40. Kees MG, Minichmayr IK, Moritz S, Beck S, Wicha SG, Kees F, Kloft C, Steinke T. Population pharmacokinetics of meropenem during continuous infusion in surgical ICU patients. J Clin Pharmacol. 2016;56(3):307-15.

41. Taccone FS, Hites M, Beumier M, Scolletta S, Jacobs F. Appropriate antibiotic dosage levels in the treatment of severe sepsis and septic shock. Curr Infect Dis Rep. 2011;13(5):406-15.

42. Tam VH, McKinnon PS, Akins RL, Rybak MJ, Drusano GL. Pharmacodynamics of cefepime in patients with Gram-negative infections. J Antimicrob Chemother. 2002;50(3):425-8.
43. Manduru M, Mihm LB, White RL, Friedrich LV, Flume PA, Bosso JA. In vitro pharmacodynamics of ceftazidime against Pseudomonas aeruginosa isolates from cystic fibrosis patients. Antimicrob Agents Chemother. 1997;41(9):2053-6.

44. Mouton JW, den Hollander JG. Killing of Pseudomonas aeruginosa during continuous and intermittent infusion of ceftazidime in an in vitro pharmacokinetic model. Antimicrob Agents Chemother. 1994;38(5):931-6.

45. Lodise TP, Sorgel F, Melnick D, Mason B, Kinzig M, Drusano GL. Penetration of meropenem into epithelial lining fluid of patients with ventilator-associated pneumonia. Antimicrob Agents Chemother. 2011;55(4):1606-10.

46. Byl B, Jacobs F, Roucloux I, de Franquen P, Cappello M, Thys JP. Penetration of meropenem in lung, bronchial mucosa, and pleural tissues. Antimicrob Agents Chemother. 1999;43(3):681-2.

47. Mader MM, Czorlich P, Konig C, Fuhrmann V, Kluge S, Westphal M, Grensemann J. Intrathecal penetration of meropenem and vancomycin administered by continuous infusion in patients suffering from ventriculitis-a retrospective analysis. Acta Neurochir. 2018;160(11):2099-105.

48. Imani S, Buscher H, Marriott D, Gentili S, Sandaradura I. Too much of a good thing: a retrospective study of beta-lactam concentration-toxicity relationships. J Antimicrob Chemother. 2017:72(10):2891-7.

49. Fantin B, Farinotti R, Thabaut A, Carbon C. Conditions for the emergence of resistance to cefpirome and ceftazidime in experimental endocarditis due to Pseudomonas aeruginosa. J Antimicrob Chemother. 1994;33(3):563-9.

50. Day T, Read AF. Does high-dose antimicrobial chemotherapy prevent the evolution of resistance? PLoS Comput Biol. 2016;12(1):e1004689.

51. Gugel J, Dos Santos Pereira A, Pignatari AC, Gales AC. Beta-Lactam MICs correlate poorly with mutant prevention concentrations for clinical isolates of Acinetobacter spp. and Pseudomonas aeruginosa. Antimicrob Agents Chemother. 2006;50(6):2276-7.

52. Rhodes NJ, Kuti JL, Nicolau DP, Van Wart S, Nicasio AM, Liu J, Lee BJ, Neely $\mathrm{MN}$, Scheetz MH. Defining clinical exposures of cefepime for gram-negative bloodstream infections that are associated with improved survival. Antimicrob Agents Chemother. 2015;60(3):1401-10.

53. Ehmann L, Zoller M, Minichmayr IK, Scharf C, Maier B, Schmitt MV, Hartung $N$, Huisinga W, Vogeser M, Frey L, et al. Role of renal function in risk assessment of target non-attainment after standard dosing of meropenem in critically ill patients: a prospective observational study. Crit Care. 2017;21(1):263.

54. Heil EL, Nicolau DP, Farkas A, Roberts JA, Thom KA. Pharmacodynamic target attainment for cefepime, meropenem, and piperacillin-tazobactam using a Pharmacokinetic/Pharmacodynamic-based dosing calculator in critically III patients. Antimicrob Agents Chemother. 2018;62(9):e01008-18.

\section{Publisher's Note}

Springer Nature remains neutral with regard to jurisdictional claims in published maps and institutional affiliations.

\section{Submit your manuscript to a SpringerOpen ${ }^{\circ}$ journal and benefit from:}

- Convenient online submission

- Rigorous peer review

- Open access: articles freely available online

- High visibility within the field

- Retaining the copyright to your article

Submit your next manuscript at springeropen.com 